\title{
Ansiedad, estrés, y estados de ánimo del Personal Especialista en Extinción de Incendios Forestales Anxiety, stress, and mood states of wildland firefighters
}

Fabio García-Heras Hernández, Jorge Gutiérrez Arroyo, O Iga M olinero González Universidad de León (España)

\begin{abstract}
Resumen: Con la presente investigación se busca principalmente establecer un perfil de ansiedad, estrés y estados de ánimo del Personal Especialista en Extinción de Incendios Forestales (PEEIF) o bomberos forestales tanto a nivel general como en situaciones de emergencia. La muestra se compuso de 24 sujetos a los que se administró una batería de cuestionarios en tres ocasiones (sin emergencia, primera emergencia y última emergencia), compuesta por un cuestionario sociodemográfico adhoc, el Perfil de Estados de Ánimo (PO M S); y el Inventario de ansiedad estado-rasgo (STAI- E/ R), realizando un análisis descriptivo y comparativo a través de la prueba t-student y comparaciones múltiples con Anova de medidas repetidas. Se observó que los miembros PEEIF, a nivel general, tenían val ores bajos de ansiedad estado, ansiedad rasgo, tensión, depresión, cólera, fatiga y confusión, mientras que el vigor fue elevado. En situaciones de emergencia, los val ores se vieron modificados en su totalidad, debido a la fuente de estrés que supone. Se demuestra que el trabajo de extinción de incendios forestales se caracteriza por tener efectos negativos a nivel emocional sobre sus profesionales, mediados por la experiencia, cargo y condiciones familiares.
\end{abstract}

Palabras clave: Ansiedad, estrés, estados de ánimo, PEEIF, bomberos forestales.

\begin{abstract}
The present research mainly seeks to establish a profile of anxiety, stress and moods of the Forest Fire Extinction Special ist Personnel (PEEIF) or wildland firefighter sboth at a general level and in emergency situations. The sample was made up of 24 subjects who were administered a battery of questionnaires on three occasions (no emergency, first emergency and last emergency), composed of an ad-hoc sociodemographic questionnaire, the Profile of M ood States (PO M S); and the StateTrait Anxiety Inventory (STAI-E / R), car rying out a descriptive and comparative analysis through the bat-student test and multiple comparisons with repeated measures Anova. It was observed that the PEEIF members, at a general level, had low values of state anxiety, trait anxiety, tension, depression, anger, fatigue and confusion, while vigor was high. In emergency situations, the values were modified in their entirety, due to the source of stress involved. It is shown that forest fire extinguishing work ischaracterized by having negative emotional effects on its professionals, mediated by experience, position and family conditions.
\end{abstract}

Key words: Anxiety, stress, mood states, PEEIF, wildland firefighters.

\section{Introducción}

El incremento de la gravedad de los incendiosforestales (IIFF), provoca, además del aumento de pérdidas ambientales y económicas, cada vez un mayor esfuerzo humano y material en su extinción y supone un mayor impacto en el personal destinado a este fin (Bowman et al., 2017). La ardua tarea que supone la extinción de IIFF recae en España y en concreto en el N oroeste de la Península sobre distintas brigadas, las cuales denomina remos como Personal Especialista en Extinción de Incendios Forestales (PEEIF). La labor profesional de estas brigadas se constata como un desempeño eminentemente físico muy exigente (Rodríguez-M arroyo et al.,

Fecha recepción: 23-10-20. Fechade aceptación: 16-12-20

Fabio García-Heras Hernández

fgarh@unileon.es
2011), en la que las condiciones ambientales (temperatura, duración del incendio, humedad, orografía, exposición al calor, altainhalación de humos, el peso del equipo de protección, dificultad de avituallamiento, etc. ), junto con las condiciones comportamentales (estrés emocional, toma de decisiones, nerviosismo, etc. ), conllevan al PEEIF a una carga física y mental extremada mente elevada (Carballo-Leyenda, Villa, López-Satué, \& Rodríguez-M arroyo, 2019; Parker, Vitalis, Walker, Riley, \& Pearce, 2017; Vidal, Fernández, \& Linari, 2007). Dada la diversidad, la naturaleza desafiante de sus responsabilidades laborales, y la exigencia física que se les demanda, es de esperar que este tipo de trabajadores lleven a cabo un entrenamiento físico y mental regular con el objetivo de mantener niveles elevados de condición física, y mejorar la relación con la salud y seguridad laboral (Rodríguez-Marroyo et al., 2012). La alta exigencia física y mental que genera la emergencia conlle- 
va elevados niveles de ansiedad y estrés sobre este tipo de personal (Aisbett $\&$ Nichols, 2007; Carleton et al., 2020; Chen, Zhang, Peng, \& Chen, 2020; Piñar-Navarro, Fuente, González-Jiménez, \& Hueso-M ontoro, 2020; Robinson, Leach, 0 wen-Lynch, \& Sünram-Lea, 2013).

Márquez, dedujo a partir de la observación de múltiples definiciones de ansiedad, que se trata de un esta do especial de agitación y tensión, con reacciones somáticasy psíquicas especial es por anticipación, recuerdo 0 experimentación actual de situaciones de inseguridad 0 amenaza, tanto real como imaginaria ( $M$ árquez, 2004), y es con esta definición con la que nos vamos a quedar, ya que recoge la multidimensionalidad del concepto, así como la variabilidad entre los sujetos que la experimentan. Por otro lado, se puede definir el estrés como un proceso que incluye la percepción de un desequilibrio sustancial entre las demandas ambientales y la capacidad de respuesta, y donde se percibe que un fallo en la satisfacción de esas demandas, tendrá consecuencias importantes, lo que produce un aumento de losniveles deansiedad (M artens, Vealey, \& Burton, 1990). 0 tro autor de referencia (Spielberger, 1976), realiza una conceptualización diferente del estrés, y sugiere que el término se reserve exclusivamente para referirse a la magnitud del peligro objetivo asociado con las propiedades de estimulación de una situación determinada, es decir, aquellas condiciones 0 circunstancias ambientales que se caractericen por la presencia de algún gra do de peligro físico o psicológico objetivo. Esto puede relacionarse con una respuesta del organismo, que tiene diversos componentes: psicológico (irritabilidad, ansiedad, etc.); comportamental (temores, pérdida de sueño, etc.); fisiológico (incremento de la FC, tensión muscular, etc. ), incluso episodios muy leves de ansiedad/ estrés se asocian a menudo con disminuciones en la efectividad en el trabajo (Molinero, Salguero, \& Márquez, 2012). El término estresor hace referencia a cualquier amenaza real o percibida por el sujeto, capaz de desorganizar el equilibrio orgánico y anímico del mismo. Los factores estresantes en los IIFF son diversos, aunque se podrán dividir en dos: fisiológicos, que incluye esfuerzo físico extremo, deshidratación, hipoglucemia, etc.; y cognitivos, que abarcarían todas aquellas amenazas 0 estresores, y que pueden producir consecuencias en el sujeto como miedo 0 ansiedad (Vidal et al., 2007). Para combatirlos, se establece el trabajo de técnicas de autocontrol (conocimiento del material y protocolos; prácticas de autocontrol y desensibilización a situaciones de riesgo, etc.) (Rotger, 1994), estrategias de afrontamiento (Angehrn, Krakauer, \& Carleton, 2020; Lope Fernández \& Solís Briceño, 2020; Piñar-N avarro et al., 2020) y en especial hacer hincapié en el mantenimiento de una buena forma física, como partes funda mentales (Araque-M artínez, Ruiz-M ontero, \& ArtésRodríguez, 2020; Rotger, 1994).

Diversos autores han indicado cómo los equipos de primera intervención en emergencias, dentro de los cuales encontramos a los PEEIF, son susceptibles de convertirse en víctimas de un acontecimiento traumático como consecuencia del desempeño de su trabajo (Angehrn et al., 2020; Curilem, Almagià, Yuing, \& Rodríguez, 2014; Leal, Villoria, M artín, Paso, \& Bulbena, 2008; Rotger, 1994; Ruiz \& Guerrero, 2017; Senabre, 2006). En un estudio que anal izó uno de los peores incendios en España, ocurrido en julio de 2005 en Riba de Saelices, (Guadalajara), en el que 11 PEEIF perdieron la vida, se establece que la sintomatología más frecuente se relacionaba con la ansiedad e hiperactivación general del organismo, manifestando sentimientos de rabia, tristeza, impotencia, frustración y desaprobación de la actitud de sus superiores (Senabre, 2006).

0 tros estudios relacionados sobre bomberos urbanos, indican que en el desempeño de su actividad laboral, se vean sometidos a altas cargas de estrés físico y emocional, apareciendo miedos y fobias provocadas por el peligro de muerte para uno mismo, actividades temerarias de compañeros y la posible incompatibilidad entre compañeros de turno (Carleton et al., 2020; Chen et al., 2020; Curilem et al., 2014; Leal et al., 2008; Piñar-N avarro et al., 2020). Según Leal et al. (2008) el colectivo de emergencias es una población con riesgo de padecer daños físicos y psíquicos debidos a su alta vulnerabilidad, a factores de estrés y a sus posibles consecuencias, lo que se refleja entre otros, en un mayor número de jubilaciones anticipadas, un mayor absentismo laboral y elevado consumo de tóxicos, determinando que a menudo no logren hacer frente al estrés en su trabajo diario (Chen et al., 2020; Wagner, Heinrichs, \& Ehlert, 1998). Un estudio realizado a 101 bomberos voluntarios de entre 32 y 42 años en Valparaiso y Viña del $M$ ar (Chile), señala que el estrés mental de su actividad puede desembocar en el Síndrome de Burnout, una respuesta al estrés laboral crónico, que conlleva en un estado emocional de agotamiento permanente, desarrollando fatiga, desgaste emocional, incapacidad de concentración, ansiedad, insomnio e irritabilidad (Curilem et al., 2014). El riesgo psicológico ante situa ciones de catástrofe y rescate, observado en profesiona les (bomberos, policías y emergencias sanitarias) que 
actuaron en diferentes tragedias como el 11M en $\mathrm{Ma}$ drid, 11S, en NuevaYork, etc. indica que aquellas condiciones de trabajo extremas, pueden producir a largo plazo una serie de reacciones cognitivas, conductuales, emocionales y físicas, que afectan tanto a la realización de la tarea, como posteriormente a la reincorporación a la vida familiar, laboral y social (Aguayo et al., 2016; Leal et al., 2008).

A partir de lo acontecido, se ha considerado el estudio de factores psicológicos en el PEEIF, ya que las condiciones a las que continuamente están expuestos, pueden generar al tos niveles de ansiedad, provocando respuestas peligrosas para la integridad del sujeto y la de sus compañeros (Vidal et al., 2007). Se establece la hipótesis de que el perfil emocional del PEEIF se modifica en situaciones de emergencia y como estas situaciones pueden tener efectos negativos sobre los profesionales del sector. Así mismo se estableció como objetivo principal de la presente investigación, establecer un perfil de ansiedad y estados de ánimo de los PEEIF españoles tanto a nivel general, como en situaciones de emergencia, para posteriormente observar la variación de la ansiedad estado, ansiedad rasgo y estados de ánimo a lo largo de la campaña de IIFF. Por ello, se pretende mostrar las diferencias existentes en función de distintas variables independientes; y comprobar si existe rela ción que pudiera derivar en la posible disminución del rendimiento laboral.

\section{M étodo}

\section{Sujetos}

En la presente investigación participó un Equipo Integral de Lucha contra Incendios Forestales (ELIF) durante la Campaña de Extinción de Incendios Forestales 2016, en concreto una unidad helitransportadade PEEIF $(n=24)$ la cual estaba dividida en tres turnos o cuadrillas junto con un técnico de operaciones y telecomunicaciones. Cada cuadrilla compuesta por seis especialistas, un mando medio o capataz y un mando superior o técnico. Las ELIF se caracterizan por la rápida actuación en sus intervenciones donde son guiados por técnicos cualificados para conseguir la extinción del mismo en el menor tiempo posible, mediante el ataque directo con herramientas manuales específicas, y siempre manteniendo las normas de seguridad, establecidas por el organismo competente (INFOCAL, Incendios Forestales de Castilla y León). En los periodos sin emergencia deben llevar a cabo entrenamiento físico, práctico y de comportamiento del incendio para así estar preparados y en las mejores condiciones de hacer frente a la emergencia. En la Tabla 1 (variables de la muestra), se pueden observar las particularidades de la misma.

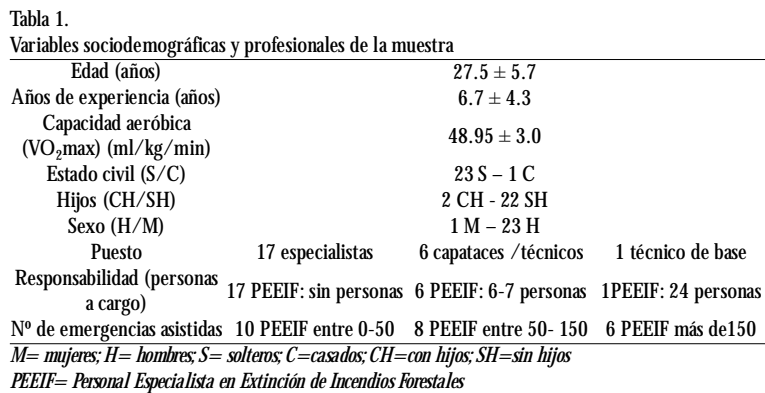

\section{Instrumentos}

Se administró una batería compuesta por tres cuestionarios: un cuestionario sociodemográfico elaborado adhoc para el presente estudio, el Cuestionario de Ansiedad Estado y Rasgo (STAI- R y STAI- E), y el Perfil de Estados de Ánimo (POMS):

- Cuestionario Sociodemográfico para profesionales en IIFF: Dicho cuestionario consta de 16 ítems en el que se tendrán en cuenta variables sociodemográficas (edad, sexo, estado civil, etc. ), profesionales y aquellas varia bles relativas a la formación en general y en la extinción de incendios forestales (años de experiencia y motivos de elección de la profesión, etc.). Además, se llevó a cabo el test Coursse $N$ avette, test de valoración de la resistencia aeróbica, de carácter indirecto, progresivo, continuo y maximal, que estima el VO ${ }_{2}$ máx en función del número de recorridos de ida y vuelta sobre una distancia de $20 \mathrm{~m}$ efectuados sobre una pista. La velocidad de la prueba aumenta progresivamente y termina cuando el sujeto no logra llegar a tiempo a la línea de $20 \mathrm{~m}$ dos veces seguidas. U na vez realizado el protocolo se recoge la velocidad alcanzada y la edad, y así estimar con una validez del . 84 el V0 2 max (Gadoury \& Léger, 1986).

- Inventario de ansiedad estado rasgo «State-Trait Anxiety Inventory» (STAI- E/ R.): Evaluación de la ansiedad mediante el Cuestionario de Ansiedad Estado/ Rasgo - STAI. (Spielberger, Gorsuch, \& Lushene, 2015) Este instrumento permite la autoevaluación de la ansiedad mediante dos conceptos de la misma: predisposición a la ansiedad (ansiedad rasgo -AR), y nivel transitorio de ansiedad en el momento presente (ansiedad estado $A E)$. Consta de 40 elementos sobre una escala de respuesta con cuatro puntos, que va desde cero a tres, y proporciona una puntuación de ansiedad rasgo y otra de ansiedad estado. Valores deAE y deAR mayor 0 igual a 45 suponen niveles altos, entre 30 y 44 niveles medios, y menores o iguales a 30 niveles bajos de AE y AR 
(González, Martín, Grau, \& Lorenzo, 2007).

- Perfil de Estados de Ánimo - «Profile of Mood States» (POMS). Es uno de los instrumentos más utilizados en psicología para la medición de los estados de ánimo. En su forma original estaba formado por una lista de 65 adjetivos, que originariamente fueron desarrollados por McN air, Loor y Droppleman en 1971 (Molinero et al., 2012), siendo utilizado como método de evaluación en diferentes poblaciones, como deportistas (España-Romero et al., 2009). Se establecen siete variables del presente cuestionario las cuales son: tensión, depresión (DEPR.), cólera, vigor, fatiga, confusión (CONF.) y alteración emocional total (A.E.T.). El perfil de estados de ánimo obtenido por los que practicaban deporte presentaba las siguientes características: valores más bajos que los no deportistas en tensión, depresión, cólera, fa tiga y confusión y altos valores en vigor. Más adelante M organ describió esta configuración como el «perfil iceberg» (Balaguer, Fuentes, García-M erita, Pérez-Recio, \& Meliá, 1993).

\section{Procedimientos}

Tras la explicación de las características del estudio a 24 PEEIF, todos ellos firmaron un consentimiento informado por escrito a través del cual se comprometían a participar voluntariamente en la investigación. La Batería de Cuestionarios fue administrada a lo largo de la campaña de incendios forestal es de 2016 en tres momentos distintos. La toma uno se real izó a principio de la temporada (entre los días 4 y 5 de julio) a modo de evaluación inicial (sin emergencia), y posteriormente se realizaron dos administraciones más (posterior a la emergencia), una correspondiente al primer incendio forestal de la campaña (toma dos), y la última administración correspondió al último incendio de la campaña de extinción (toma tres). La administración de los cuestionarios post intervención se realizó en los minutos posteriores al aterrizaje en base, sin despojarse el Equipo de Protección Individual, y permitiendo un aseo bá sico (menor a dos minutos). Una vez recogidos todos los datos se procedió a su codificación y elaboración de la correspondiente base de datos para su posterior aná lisis.

\section{Análisis estadísticos}

En primer lugar, se llevó a cabo un análisis descriptivo calculando medias y desviación típica de cada una de las variables analizadas. Posteriormente se llevó a cabo un análisis comparativo con la pruebaT para muestras independientes (dos variables) y un Anova de me- didas repetidas para comparar las distintas tomas y comprobar las diferencias entre grupos en función de las distintas variables independientes, en aquellas con más de dos variables. A la hora de obtener la significación, si la probabilidad asociada es menor 0 igual .05 suponemos varianzas distintas. El nivel de significación que se tuvo en cuenta fue de.05. Se utilizó el programa estadístico SPSS 21.0 paraW indows, y para el tratamiento gráfico se empleó el programa M icrosoft O ffice Excell 2007.

\section{Resultados}

Perfil general y en emergencias de los PEEIF

A continuación, mostramos los datos descriptivos, en primer lugar, características generales (medición previa o toma uno) del PEEIF (Tabla2), y en segundo, el perfil de emergencias (Tabla 3), estableciendo que tienen niveles de $A E$ y $A R$ similares. En relación a los estados de ánimo destacar el bajo nivel en confusión, tensión y fatiga, y un alto índice de vigor. A su vez, en la Figura 1, se muestra el perfil Iceberg evaluado por el POMS, del PEEIF, a nivel general y durante diferentes emergencias.
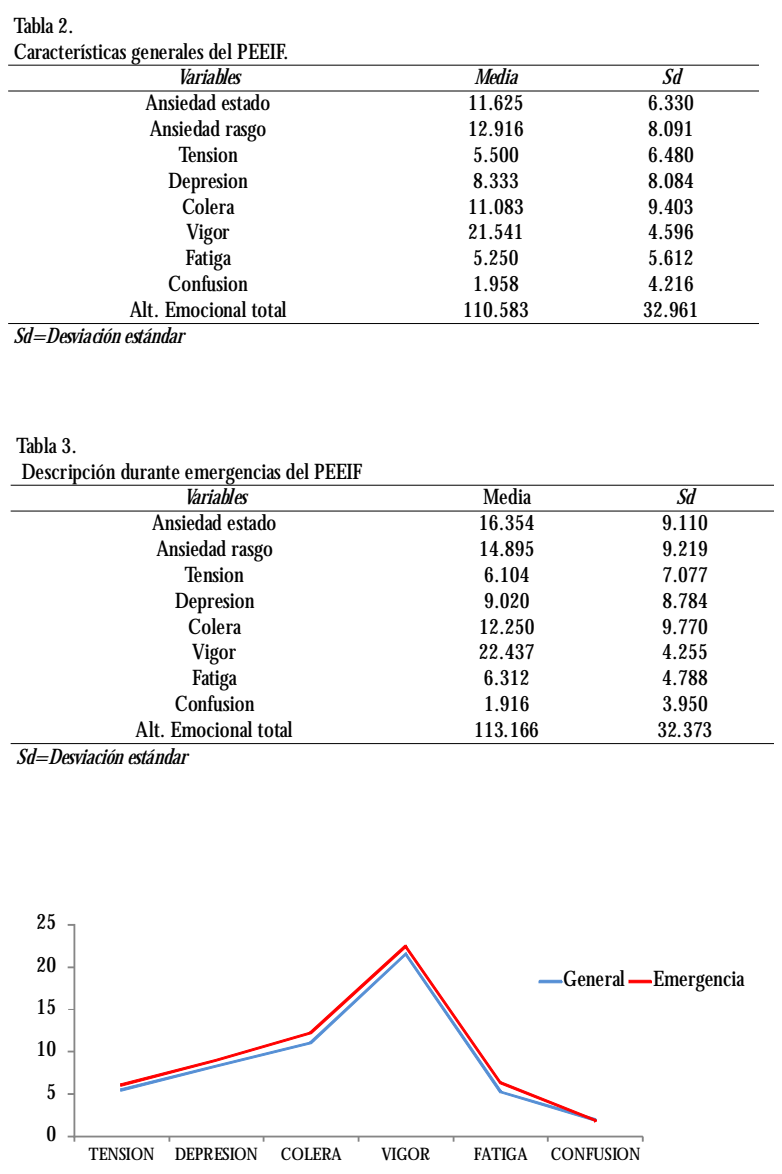

Figura 1: Perfil iceberg evaluado por el POMS, del PEEIF

- Comparación del perfil iceberg del PEEIF, enfrentando los valores a nivel general y en emergencia- 
Variación de la ansiedad estado, ansiedad rasgo y estados de ánimo a lo largo de la campaña de IIFF

Como muestra laTabla 4 a lo largo de la campaña de IIFF, se ha observado como los valores de AE y AR, han sufrido un aumento. Así mismo, los valores que conforman el perfil de estados de ánimo tienen valores inferiores en la toma uno (sin emergencia) y tres (última emergencia), observándose los mayores valores en la toma dos (primera emergencia de la campaña), sal vo en fatiga y confusión, donde los mayores valores se observan en la toma tres (última emergencia de la temporada de IIFF). Al hacer comparaciones múltiples, se observan diferencias significativas en $A E$, entre toma uno y tres $\left(p=.012^{*}\right)$. En el resto de valores no son apreciables diferencias estadísticamente significativas.

Tabla 4.

Valores ansiedad estado/ rasgo, y perfil de estados de ánimo durante la campaña de extinción de IIFF

\begin{tabular}{|c|c|c|c|c|c|c|c|c|c|c|}
\hline \multicolumn{2}{|c|}{ TOMA } & $\mathrm{AE}$ & AR & TENSION & DEPR. & COLERA & VIGOR & FATIGA & CONF. & A.E.T \\
\hline \multirow[b]{2}{*}{1} & Media & 11.62 & 12.91 & 5.50 & 8.33 & 11.08 & 21.5 & 5.25 & 1.95 & 110.55 \\
\hline & $S d$ & 6.33 & 8.09 & 6.48 & 8.08 & 9.40 & 4.59 & 5.61 & 4.21 & 32.96 \\
\hline \multirow[b]{2}{*}{2} & Media & 14.91 & 14.33 & 6.62 & 10.20 & 13.0 & 23.25 & 6.20 & 1.75 & 114.54 \\
\hline & $S d$ & 8.47 & 8.74 & 7.87 & 9.84 & 10.37 & 3.57 & 4.45 & 4.05 & 33.91 \\
\hline \multirow{2}{*}{3} & Media & 17.83 & 15.45 & 5.58 & 7.83 & 11.50 & 21.62 & 6.41 & 2.08 & 111.79 \\
\hline & $S d$ & 9.69 & 8.35 & 6.27 & 7.72 & 9.16 & 4.93 & 5.12 & 3.84 & 30.83 \\
\hline \multirow{3}{*}{ Total } & Media & 14.79 & 14.23 & 5.90 & 8.79 & 11.86 & 22.13 & 5.95 & 1.93 & 112.30 \\
\hline & $S d$ & 8.55 & 8.34 & 6.83 & 8.54 & 9.56 & 4.41 & 5.03 & 3.98 & 32.17 \\
\hline & p & c & n.s. & n.S. & n.S. & n. S. & n.S. & n.S. & n.s. & n.s. \\
\hline
\end{tabular}

a. Diferencias significativas entre toma 1 y toma 2. b. Diferencias significativas entre toma 2 y toma 3. C. Diferencias significativas entre toma 1 y toma 3 ; Diferencias significativas $(p=\langle .05)$; $n$.s.: diferencias no significativas; Sd: desviación estándar, AE: ansiedad estado; AR: ansiedad rasgo; DEPR: depresión; CON F: confusión; A. E.T.: Alteración emocional total

\section{Diferencias existentes en las variables de an-} siedad estado, rasgo y estados de ánimo en función de distintas variables independientes

En este apartado se van a analizar diferentes varia bles independientes, que, según la bibliografía revisada y el propio criterio, se han considerado claves. Las va riables se han dividido en tres: personales (hijos y esta do civil), fisiológicas (VO 2max), y laborales (puesto de trabajo y $n-$ de emergencias asistidas). Con las variables dependientes de $A E, A R$, tensión, depresión, cólera, vigor, fatiga, confusión y alteración emocional total.

Primeramente, en la variable hijos y estado civil (Tabla 5), se observa como los que no tienen descendencia, tienen valores más elevados en $A E, A R$, tensión, depresión, cólera, fatiga y alteración emocional total, salvo en el vigor que lo tienen menor. Cabe destacar que las diferencias son significativas a lo largo de las tres tomas, ya que $p<.05$, en todos los ítems, salvo en vigor, que $p>.05(p=.114)$. Así mismo son los PEEIF solteros ( $95.8 \%$ de la muestra) tienen valores mayores en todas las dependientes, salvo en vigor, que es menor. Por el contrario, los sujetos casados $(4.2 \%)$ tienen menores valores en todas las variables depen- dientes, salvo en vigor, que es mayor. Las diferencias son significativas en las siguientes variables dependientes: $A E(p=.000), A R(p=.000)$, tensión $(p=.000)$, depresión $(p=.000)$, cólera $(p=.000)$, vigor $(p=$ $.040)$, fatiga $(p=.000)$, confusión $(p=.000)$, y altera ción emocional total $(p=.000)$.

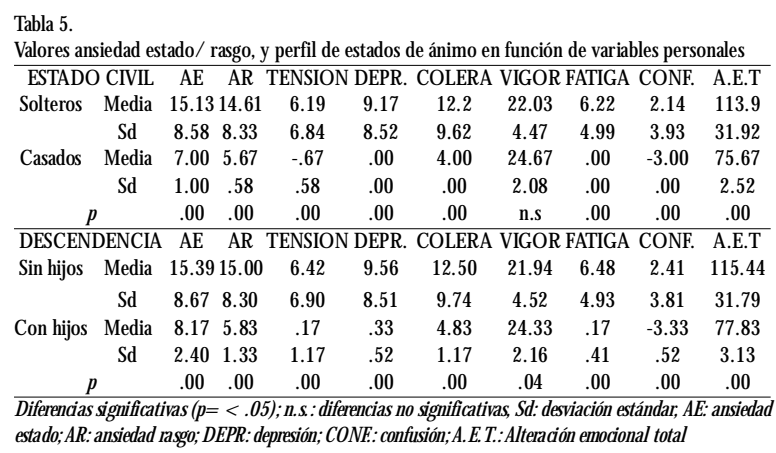

Respecto al $\mathrm{VO}_{2}$ max, el cual se ha dividido en aquellos sujetos con valores por encima de la media (M ean $\mathrm{VO}_{2} \max =48.95 \mathrm{ml} / \mathrm{kg} / \mathrm{min}$ ) y aquellos por debajo de la media (Tabla 6), los datos establecen que aquellos sujetos con mejor forma física, tienen valores más elevados en todas las variables dependientes, respecto a los sujetos que están por debajo de la media.

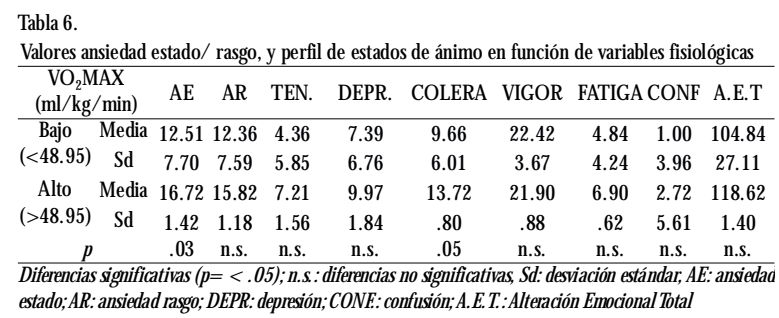

La variable independiente de Puesto deTrabajo (Ta bla 7), la cual está dividida en: peones (sin personas a su cargo), capataces (seisper sonasasu cargo), técnicos(siete 0 más personas a su cargo), y técnico de base (responsable de 24 personas). Se observa en AE, AR, tensión, depresión, confusión yA.E.T. Mayoresvalores para sujetos con mayor responsabilidad, técnicos de base y técnicos. Por otro lado, en las variables cólera, vigor y fatiga, peones y capataces tienen los mayores valores. Las diferencias significativas aparecen al comparar to- 
dos los puestos de trabajo en las variables que aparecen en laTabla 7.

Para terminar, analizamos la variable independiente «número de emergencias asistidas durante su vida laboral» (Tabla 8). Aquellos sujetos con menor cantidad de emergencias, o lo que es lo mismo, con menor experiencia, tienen valores mayores en cuanto a las variables dependientes se refiere, salvo en AE y cólera, que los sujetos con mayor experiencia, cuentan con unos valores ligeramente superiores, y en vigor, mayores valores para aquellos con mayor experiencia.

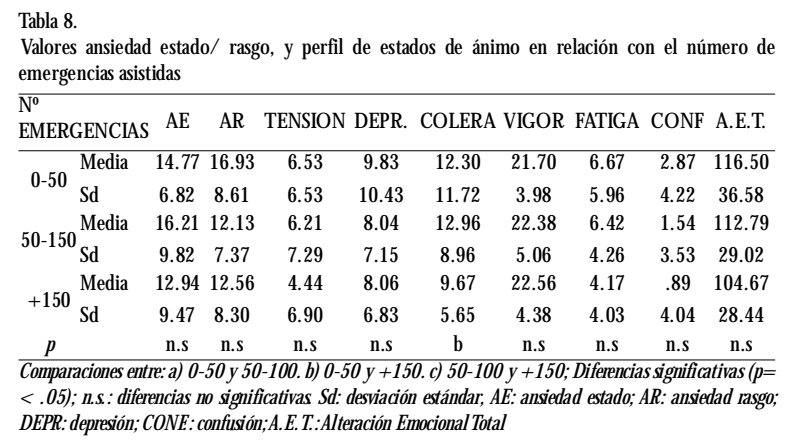

Valores ansiedad estado/ rasgo, y perfil de estados de ánimo en relación con el número de emergencias asistidas

No
EMERGENCIAS AE AR TENSION DEPR. COLERA VIGOR FATIGA CONF A.E.T.

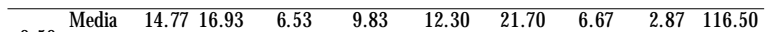
$\begin{array}{lllllllllll}0-50 & \text { Sd } & 6.82 & 8.61 & 6.53 & 10.43 & 11.72 & 3.98 & 5.96 & 4.22 & 36.58\end{array}$

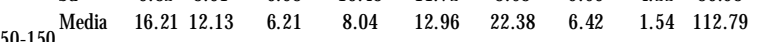

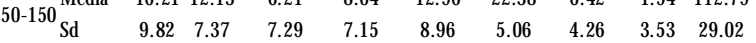
$\begin{array}{llllllllll}\text { Media } & 12.94 & 12.56 & 4.44 & 8.06 & 9.67 & 22.56 & 4.17 & .89 & 104.67\end{array}$

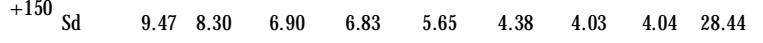

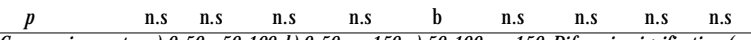
$<.05$ ); n.s.: diferencias no significativas Sd: desviación estándar, AE: ansiedad estado; AR: ansiedad rasgo; DEPR: depresión; CON F.: confusión; A. E.T.:Alteración Emocional Total

\section{Discusión}

EI PEEIF que ha participado en este estudio se trata de una muestra de profesionales que trabajan en el Noroeste de la Península Ibérica, en concreto dentro del Parque N atural de Sanabria, Zamora, una zona geográ fica que tiene una tasa superior de emergencias de IIFF en comparación con la media nacional, superada entre otras por Galicia y la comarca del Bierzo en León. Hasta donde sabemos es el primer estudio que ha anal izado variables psicológicas en el PEEIF, como se modifican tras una emergencia y su evolución durante la campaña de extinción de IIFF.

A nivel general, el PEEIF tiene niveles bajos de $A E$ yAR, ya que los valores más elevados obtenidos tanto a nivel general $(11.62 \pm 6.3,12.9 \pm 8.1, A E$ y AR respectivamente) como en emergencia ( $16.3 \pm 9.1$ para AE y $14.9 \pm 9.2$ en AR) no superan los valores de referencia (alto e» 45, medio 30-44, y valores bajos < 30) (González et al., 2007) . Así mismo, se puede decir que se encuentran por debajo de los valores promedio de la población española (Buela-Casal \& Guillén-Riquelme, 2017). Por otro lado, en un estudio realizado a 49 bomberos urbanos de la ciudad de Loja- Ecuador, establece que alrededor del $85 \%$ de los Bomberos presentan niveles de ansiedad de carácter muy leve (Jaramillo-Vicente, 2013). Entre otras cuestiones, niveles tan bajos deAE yAR pueden ser provocados por los niveles ele- vados deVO $\mathrm{max}_{2}$ de la muestra y la realización de actividad física de manera regular durante la campaña de IIFF, ya que está descrito que la práctica de actividad física de una manera regular se correlaciona con menoresvaloresde ansiedad (Ackerknecht \& M endoza, 2007; Araque-M artínez et al., 2020; M cAuley, Marquez, Jerome, Blissmer, \& Katula, 2002; O Imedilla-Zafra \& OrtegaToro, 2009).

En cuanto al Perfil de Estados de Ánimo, el PEEIF, al igual que diferente población de deportistas de élite muestra el denominado perfil Icerberg (Andrade, Fernández, \& Pesqueira, 2002; Balaguer, Escartí, \& Villamarín, 1995; M olinero et al., 2012). El PEEIF durante el desempeño de su trabajo tiene niveles de carga física similares a distinta población deportista de alto nivel como corredores de media maratón, maratón, y ciclistas, entre otros (Rodríguez-M arroyo et al. , 2011). Es por ello que se ha considerado comparar nuestros datos con los obtenidos de una muestra de 194 deportistas de diversas disciplinas a nivel competitivo (González-Boto, Salguero, Tuero, \& Márquez, 2009) (Figura 2), el PEEIF desarrolla niveles inferiores en las variables negativas del POMS y del STAI E/ R, mientras que el vigor se muestra por encima, estableciéndose claramente el anteriormente citado «Perfil Iceberg».

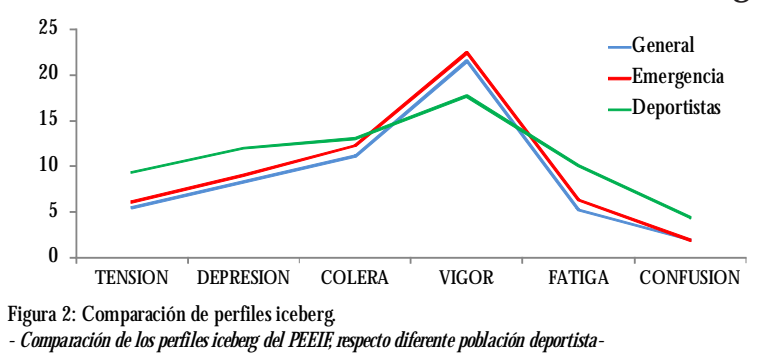

La utilización del POMS ha permitido confirmar los beneficios psicológicos de la participación en programas de ejercicio, observándose que sus puntuaciones varían con la intensidad del entrenamiento y se ha sugerido que este instrumento podría utilizarse para la monitorización del sobreentrenamiento (Martin, Andersen, \& Gates, 2000; Molinero et al., 2012). La introducción de este tipo de cuestionarios junto con una programación pautada de ejercicio físico dentro de la jornada del PEEIF va a permitir comprobar la carga tanto física como mental y monitorizar la fatiga crónica y la optimización del entrenamiento.

O bservando la variación de las variables independientes a lo largo de la campaña de IIFF, se puede decir que laAE (11.62 $\pm 6.3 ; 16.3 \pm 9.1$ valores iniciales, y valores finales de la campaña) y laAR (12.9 \pm 8.1 datos iniciales; $14.9 \pm 9.2$ finales) ha ido en aumento, donde 
los mayores valores se han obtenido en la toma tres, correspondiendo con laúltimaemergencia evaluada, que se caracterizó por el desconcierto, altas horas de traba jo, malestar general, y poca satisfacción con el trabajo realizado. Estos datos, al igual que los obtenidos en un estudio de una U nidad del Ejército enYugoslavia (Pérez \& Rodríguez, 2011), en el que se les administró el STAI E/ R, antes de acudir $(20.12 \pm 17.9 ; 18.53 \pm 19.8 \mathrm{AE}$ y $A R$ respectivamente) y posteriormente en la zona de operaciones ( $28.55 \pm 26.1 ; 19.35 \pm 24.5 \mathrm{AE}$ y AR respectivamente), no hacen más que apoyar que en situa ciones de emergencia, los valores de AE y AR aumen$\tan$.

Los datos obtenidos sugieren que aquellos sujetos con hijos y casados, son los que tienen menor $A E, A R, y$ menores valores en las variables del POMS. Esto se podría deber a que estos sujetos son aquellos que más años de experiencia tienen en el sector $y$, por tanto, llevan más tiempo enfrentándose a las diferentes situa ciones de emergencia. U na relación similar se ha encontrado en una muestra de 1007 maratonianos españoles de diversas edades, donde aquellos más experimentados cursaban menores valores de ansiedad (Ruiz-) uan \& Zarauz Sancho, 2015). Así mismo, se ha señalado la importancia de la experiencia en el deporte para la percepción del control de los efectos negativos de la ansiedad (Peñaloza Gómez, Jaenes Sánchez, M éndezSánchez, \& Jaenes-Amarillo, 2016).

Si bien es verdad que como forma general la rela ción entre ansiedad- rendimiento es menor en aquellos sujetos que cuentan con una preparación física mayor (Araque-Martínez et al., 2020), en la presente investigación nos encontramos una relación contraria. En rela ción al consumo de $\mathrm{O}_{2}\left(\mathrm{VO}_{2} \max \right)$, se observa que, a mayor consumo, mayores niveles de las variables dependientes, esto quiere decir que sujetos con menor capacidad æeróbica cursan con menores valores de $A E$, AR, tensión, depresión, cólera, etc. Estos datos apoyan los recogidos por (Gutiérrez- Calvo, Estévez- Monzó, García- Pérez, \& Pérez- Hernández, 1997), donde establecen que la mayor capacidad aeróbica de dos grupos de atletas evaluados, no corresponde con val ores menores de ansiedad y estrés. Consideramos que esta relación en nuestra investigación, podría ser debida a la experiencia, al número de emergencias asistidas y la edad, ya que aquellos sujetos con mejor forma física son aquellos menos experimentados y, por lo tanto, tienen menos estrategias de afrontamiento a situaciones de riesgo como es el caso de los IIFF.

Sumado a las condiciones de la propia emergencia, la mayor responsabilidad provoca que los técnicos, tengan mayores niveles en casi todas las variables evalua das (AE, AR, tensión, depresión, confusión y alteración emocional total), en este sentido una mala decisión puede provocar un accidente fatal 0 una actuación ineficaz. Un buen líder dentro de los IIFF, tiene que tener la capacidad de ser empático (con mandos, compañeros y subordinados), capacidad de comunicación, disponer de competencias emocionales, tener valores y conocimientos, y ser capaz de que todos confíen en él, y así mejorar el rendimiento del trabajo, así mismo, debe contar con la habilidad de darse cuenta de cómo se sienten sus subordinados, y de intervenir cuando se encuentren desbordados (Vidal et al., 2007).

Una de las variables principales dentro del estudio, como es la experiencia, nos muestra que el PEEIF con mayor experiencia tiene menores niveles en todas las variables dependientes salvo AE y cólera. Sin embargo esta relación, la cual se puede considerar positiva, esta blece que el PEEIF más experimentado se enfrenta a su labor de una forma más relajada y esto conlleva otro tipo de problemas, yaquesegún Ackerknecht y M endoza, (2007) en el combate contra IIFF hay una mayor tendencia a los accidentes dentro de aquellos PEEIF más experimentados, provocado por el exceso de confianza y el desafío del riesgo, amparándose en el convencimiento de que la antigüedad en la tarea le exime de la posibilidad de accidentarse.

Al igual que en nuestra investigación, donde los mayores valores de las variables dependientes cursaron con una disminución de la efectividad laboral, los mayores niveles deAE yAR provocaron una disminución del rendimiento en diversas pruebas atléticas (GutiérrezCalvo et al., 1997). Por otro lado se ha comprobado que la inclusión de programas de entrenamiento reducen los niveles de ansiedad, tensión, depresión, riesgos psicosomáticos, aportando implicaciones prácticas para la mejora de la sal ud psicológica de jóvenes deportistas (Lope Fernández \& Solís Briceño, 2020), y adultosmayores (Araque-M artínez et al., 2020), lo cual puede ayudar como forma positiva a amortiguar los efectos de la ansiedad y provocar un efecto contrario que ayude a los sujetos a una mejora en su rendimiento al reinterpretar los síntomas de ansiedad positivamente.

El presente estudio aporta datos para concluir que el trabajo de extinción de IIFF se caracteriza por tener efectos negativos a nivel emocional sobre sus profesionales, mediados especialmente por la experiencia, cargo o responsabilidad y condiciones familiares. Los valores obtenidos durante la administración de los cuestio- 
narios sin emergencia, demuestran que el PEEIF es un colectivo con niveles bajos de Ansiedad Estado, Ansiedad Rasgo, tensión, depresión, cólera, fatigay confusión respecto a la media española y el vigor se muestra claramente elevado. Durante una emergencia, se ven elevados los valores analizados siendo la variable $A E$ estadísticamente significativa. Por todo lo expuesto se debe poner de relieve la necesidad de incluir estrategias/ programas de afrontamiento de la ansiedad y de los estados de ánimo, ya que los valores antes mencionados se elevan en todos los casos, provocado especialmente por la fuente de estrés que supone la actuación en un Incendio Forestal.

\section{Aplicaciones prácticas y agradecimientos}

Consideramos que la presente investigación tiene una clara aplicación al sector de los IIFF, buscando la mejora de la actividad profesional, así como la reducción de posibles bajas laborales por estrés. Este tipo de relaciones permitiría la intervención de profesionales como psicólogos con formación y experiencia en emergencias y/ o relacionados con el deporte, preparadores físicos titulados y especializados en el sector para poder optimizar tanto este tipo de variables, como otras rela cionadas. Sería muy interesante aportar herramientas de afrontamiento de situaciones estresantes tanto a los mandos o jefes de equipo como a los propios trabajadores. En futuras investigaciones se deberá aumentar la muestra, incluyendo a otros centros a nivel nacional, y así como incluir otras variables relacionadas como el síndrome de Burnout, variables de personalidad como impulsividad, y/ o variables relacionadas con la toma de decisiones.

Durante la realización de la investigación se ha contado con la inestimable ayuda del PEEIF (compañeros y amigos), loscuales han ayudado en todo momento, aportando su paciencia, conocimientos y, en definitiva, ganas de ayudar para que el trabajo saliera adelante. No siempre es fácil ponerte a contestar un cuestionario de 104 ítems después de 12 horas en un Incendio Forestal, y ellos, sin queja alguna lo han realizado.

Agradecer la financiación del Fondo Social Europeo, Programa 0 perativo de Castilla y León, y de la Junta de Castilla y León, a través de la Consejería de Educa ción.

\section{Referencias}

Ackerknecht, C., \& M endoza, S. (2007). Estudio sobreSeguridad y Salud $O$ cupacional en el Combate de IncendiosForestalesen
Chile Introducción. Seguridad y Salud Ocupacional En Chile, 6 . Aguayo, A., López, E., Lorenzo, A., Ávila, M. E., Vera, J. A., \& Hindricks, I. (2016). Estrategias de afrontamiento anteemergencias y desastres. Cuadernos de Crisis, 5(15), 13- 20.

Aisbett, B. \& Nichols, D. (2007). Fighting fatigue whilst fighting bushfire: An overview of factors contributing to firefighter fatigue during bushfire suppression. Australian Journal of Emergency $M$ anagement, 22 (3), 31-39.

Andrade, E. M., Fernández, C. A., \& Pesqueira, G. S. (2002). Adaptaciónal español del cuestionario «Perfil de losEstadosde Ánimo» en unamuestrade deportistas. Psicothema, 14(4), 708713.

Angehrn, A. , Krakauer, R. L., \& Carleton, R. N. (2020).T helmpact of Intolerance of Uncertainty and Anxiety Sensitivity on M ental HealthAmong Public Safety Per sonnel:W hen the Uncertain is Unavoidable. Cognitive Therapy and Research, 44(5), 919930. https: / / doi.org/ 10.1007/ s10608-020-10107-2

Araque-Martínez, M. Á., Ruiz-Montero, P. J., \&Artés-Rodríguez, E. M. (2020). Efectos de un programa de ejercicio físico multicomponente sobre la condición física, la autoestima, la ansiedad y la depresión de personas adultas-mayores (Effects of a multicomponent physical exercise program on fitness, self-esteem, anxiety and depres. Retos, 2041(39), 1024- 1028. https:/ / doi.org/ 10.47197/ retos. v0i39.83282

Balaguer, I., Escartí, A., \&Villamarín, F. (1995). Autoeficaciaen el Deportey en laactividad fisica: estado actual de lainvestigacion. Revista de Psicologia Geral y Aplicada, Vol. 48, pp. 139-159.

Balaguer, I., Fuentes, I., García-M erita, M., Pérez-Recio, G., \& M eliá, J. L. (1993). El perfil delosestados deanimo (PO MS): baremo paraestudiantes valencianosy su aplicacion en el contexto deportivo. Revista de Psicología Del Deporte, 2(2), 00390052. Retrieved from http:/ / ddd. uab. cat// record/ 63162

Bowman, D. M. J. S., W illiamson, G. J., A batzoglou, J.T., Kolden, C. A., Cochrane, M. A., \& Smith, A. M. S. (2017). Human exposure and sensitivity to globally extreme wildfire events. $N$ ature Ecology and Evolution, 1(3), 1-6.

Buela-Casal, G., \& Guillén-Riquelme, A. (2017).Ver sión brevede la adaptación española del State-Trait Anxiety Inventory. International Journal of Clinical and Health Psychology, 17(3), 261-268. https:/ / doi.org/ 10.1016/ j.ijchp.2017.07.003

Carballo-Leyenda, B., Villa, J. G., López-Satué, J., \& RodríguezMarroyo, J. A. (2019). CharacterizingW ildland Firefighters' Thermal Environment During Live-FireSuppression. Frontiers in Physiology, 10(August), 1-8.

Carleton, R. N., Afifi, T. O., Taillieu, T., Turner, S., Mason, J. E., Ricciardelli, R., ... Griffiths, C.T. (2020). Assessing the relative impact of diverse stressors among public safety personnel. International Journal of Environmental Research and Public $\mathrm{H}$ ealth, 17(4). https:/ / doi.org/ 10.3390/ ijerph17041234

Chen, X., Zhang, L., Peng, Z., \& Chen, S. (2020). Factors Influencing the M ental Health of Firefighters in Shantou City, China. Psychology Research and Behavior Management, Volume13, 529-536. https:/ / doi. org/ 10.2147/ PRBM. S249650

Curilem, G. C., Almagià, F. A., Yuing, F. T., \& Rodríguez, R. F. (2014). Evaluación del estado psicobiotipológico en bomberos: Parámetros de salud y recursos anti estrés. International Journal of Morphology, 32(2), 709-714.

España-Romero,V., Artero, E. G., O rtega, F. B., Jiménez-Pavón, D., Gutiérrez,A., Castillo, M. J., \& Ruiz, J. R. (2009).A spectos 
fisiológicos de la escalada deportiva. Revista Internacional de Medicina y Ciencias de La Actividad Física y Del Deporte, 9(35), 264-299.

Gadoury, C., \& Léger, L. (1986). Validité de l'épreuve de course navette de $20 \mathrm{~m}$ avec paliers de 1 minute et du physistest canadien pour prédire leVO 2 max desadultes. Revista D esCiencias etT écnicas D esActividades Físicos et D eportivo, 7(13), 15- 28.

González-Boto, R., Sal guero, A. ,Tuero, C. , \& Márquez, S. (2009). Validez concur rente dela versión españioladel cuestionario de recuperación-estrés para deportistas (restq-sport). Revista de Psicologia D el D eporte, 18(1), 53-72.

González, M., Martín, M., Grau, J. a, \& Lorenzo, A. (2007). Instrumentos deevaluación psicológica parael estudio delaansiedad y la depresión. In Instrumentos de Evaluación Psicológica para el Estudio de la Ansiedad y la Depresión (pp. 165-214). https:/ / doi.org/ 10.13140/ RG.2.1.1029.3606

Gutiérrez- Calvo, M., Estévez- Monzó, A., García Pérez, J., \& Pérez- Hernández, H . (1997). Ansiedad y rendimiento atlético en condiciones de estrés: efectosmoduladores de la práctica. Revista de Psicología Del Deporte, 6(2), 27-46.

Jaramillo-Vicente,V. G. (2013). La incidencia del síndromedeburnout en el Cuerpo de Bomberos de Loja en el periodo junio-noviembre del 2013. Universidad Nacional de Loja.

Leal, I., Villoria, B. , Martín, L. M. , Paso, J. I. , \& Bulbena, A. (2008). A poyo psicológico desde los bomberosalas víctimasy paralos bomberos evitando que sean víctimas. Cuadernos de Crisis, 1(7), 16-22.

Lope Fernández, D. E. \& Solís Briceño, O. B. (2020). Estrategias de afrontamiento como inter vención al estrés en futbolistas (Coping strategies as a stress inter vention in soccer players). Retos, 2041(38), 613-619. https:/ / doi.org/ 10.47197/ retos.v38i38.75724

Lorenzo Ruiz,A. , \& Guerrero, A. E. (2017). Afectacionespsicológicas en personal de primerarespuesta: ¿Trastorno por estrés postraumático o estrés traumático secundario? [Psychological concerns in first responders: Post traumatic stress disorder or secondary traumatic stress?]. Revista Puertorriqueña de Psicología, 28(2), 252-265.

Márquez, S. (2004). Ansiedad, estrés y deporte. Madrid: Editorial EOS.

Martens, R. ,Vealey, R. S. , \& Burton, D. (1990). CompetitiveAnxiety in Sport. Champaign, III., USA: Human Kinetics.

Martin, D.T., Andersen, M. B. , \& Gates, W. (2000). U sing Profile of M ood States (POMS) to M onitor High-IntensityTraining in Cyclists: Group versus Case Studies. The Sport Psychologist, 14(2), 138-156. https: / / doi.org/ 10.1123/ tsp. 14.2. 138

M cAuley, E., Marquez, D. X., Jerome, G. J., Blissmer, B. , \& Katula, J. (2002). Physical activity and physique anxiety in older adults: Fitness, and efficacy influences. Aging and $M$ ental $\mathrm{H}$ ealth, 6(3), 222-230. https:/ / doi.org/ 10.1080/ 13607860220142459

M olinero, 0., Sal guero, A., \& M árquez, S. (2012). Estrés-recuperación en deportistas y su relación con los estados de ánimo y las estrategias de afrontamiento. = Stress and recovery in athletes and their relationship to mood slate disturbances and coping strategies. Revista de Psicología Del Deporte, 21 (1), 163170.

O ImedillazZafra, A., \& O rtega-Toro, E. (2009). Incidencia de la práctica de actividad física sobre la ansiedad y depresión en mujeres: perfiles de riesgo. U niversitas Psychologica, 8(1), 105-
116. Retrieved from

Parker, R., Vitalis, A., Walker, R., Riley, D., \& Pearce, H. G. (2017). Measuring wildland fire fighter performance with wearable technology. Applied Ergonomics, 59, 34- 44.

Peñaloza Gómez, R., Jaenes Sánchez, J. C., M éndez-Sánchez, M. del P., \& Jaenes-Amarillo, P. (2016). El poder explicativo dela ansiedad en los estados deánimo de deportistasespañoles(The explanatory power of anxiety in themood of Spanish athletes). Retos, 2041(30), 207-210. https:/ / doi.org/ 10.47197/ retos. v0i30.50259

Pérez, A. M ., \& Rodríguez, J. M . (2011). Análisis del estado emocional de unaunidad del ejército español en zona de operaciones. Sanidad Militar, 67(2), 71-77.

Piñar-N avarro, E., Fuente, G. A. C. D. la, González-Jiménez, E. , \& Hueso-M ontoro, C. (2020). Anxiety and strategies for coping with stress used by first responders and out-of-hospital emergency health care staff before the covid-19 pandemic. Emergencias, 32(5), 371-373.

Robinson, S. J., Leach, J ., 0 wen-Lynch, P. J. , \& Sünram-Lea, S. I. (2013). Stress reactivity and cognitive performance in a simulated firefighting emergency. Aviation Space and Environmental Medicine, 84(6), 592-599.

Rodríguez-Marroyo, J. A. , López-Satue, J., Pernía, R., Carballo, B., García-López, J., Foster, C., \& Villa, J. G. (2012). Physiological work demands of Spanish wildland firefighters during wildfire suppression. International Archives of Occupational and Environmental Health, 85(2), 221-228. https:/ / doi. org/ 10.1007/ s00420-011-0661-4

Rodríguez-M arroyo, J. A. , Villa, J. G., López-Satue, J., Pernía, R., Carballo, B., GarcíaLópez, J., \& Foster, C. (2011). Physical and thermal strain of firefighters according to the firefighting tactics used to suppress wildfires. Ergonomics, 54(11), 11011108. https: / / doi.org/ 10.1080/ 00140139.2011.611895

Rotger, D. (1994). El estrés de los bomberos. Maprfe Seguridad, (55), 11-21.

Ruiz-Juan, F. \& Z Zarauz Sancho, A. (2015). Ansiedad en maratonianos en función de variables socio-demográficas (Anxiety in marathoners based on socio-demographic variables). Retos, 2041(25), 28-31. https:// doi.org/ 10.47197/ retos.v0i25.34470

Senabre, J. (2006). La sombra de los incendios forestales. Guadalajara(España), julio de 2005: Un estudio comparativo. 4 a Conferencia Internacional Sobre Incendiios Forestales, (23), 132133.

Spiel berger, C. D. (1976). The nature and measurement of anxiety. In C. D. Spielberger \& R. Diaz-Guerrero (Eds.), Cross cultural anxiety. Washington: Hemisphere.

Spielberger, R. D., Gorsuch, R. L., \& Lushene, R. E. (2015). STAI Cuestionario deAnsiedad Estado-Rasgo. InTEA ediciones. Ma drid.

Vidal, D., Fernández, C., \& Linari, F. (2007). Laimportanciadela formación integral parala motivación, el estrés, la eficaciay laseguridad en la defensa contrafuegosforestales. IV Conferencia Internacional de Incendios Forestal esWildfire. Sevilla, España. Wagner, D., Heinrichs, M., \& Ehlert, U. (1998). Prevalence of symptoms of posttraumatic stress disorder in German professional firefighters. American Journal of Psychiatry, 155(12), 1727-1732. https:/ / doi.org/ 10.1176/ ajp. 155.12.1727 